\title{
DELEUZE Y MERLEAU-PONTY. LA CARNE DEL MUNDO
}

Gonzalo Montenegro Vargas ${ }^{2}$

\section{Resumen}

A pesar de la distancia que parecen evidenciar la filosofía de Merleau-Ponty y de Deleuze, es posible descubrir más que complicidades analógicas respecto de la crítica que dirigen a Husserl. En el terreno estético, por ejemplo, podemos constatar que las últimas reflexiones de Merleau-Ponty, presentes fundamentalmente en Lo visible y lo invisible, representan un parangón constante para el desarrollo de obras como la que Deleuze consagra al pintor Francis Bacon. Por lo tanto, la investigación que sigue se aboca a definir los lugares y motivos principales en los que, de la mano de la lectura que efectúan de Husserl, se reconocen entre Merleau-Ponty y Deleuze puntos de contacto que desafían la indiferencia aparente desde la que se comprenden sus respectivas filosofías.

\section{Palabras clave}

Deleuze, Merleau-Ponty, percepción, carne, intensidad, tiempo magma

\section{Abstract}

Despite the distance between Merleau-Ponty's and Deleuze's philosophies, it is possible to discover more than analogue complicity regarding the criticism addressed to Husserl. In the aesthetic field, for example, we notice that the last thoughts of Merleau-Ponty, present mainly in The visible and the invisible, refer constantly to works such as the one that Deleuze dedicated to the painter Francis Bacon. Therefore, this research is intended to define the main passages and motives on which, following also the reading of Husserl, we can find contact points between Merleau-Ponty and Deleuze that defy the apparently indifferent approach from which their philosophies are understood.

\section{Keywords}

Deleuze, Merleau-Ponty, perception, flesh, intensity, time magma

\section{Recibido el 17 de agosto de 2010, aprobado el 26 de agosto de 2010}

En la crítica dirigida a Husserl, Deleuze y Merleau-Ponty desarrollan un gesto similar que determinará de manera importante cierta vinculación entre estos autores a la hora de definir su postura acerca de la constitución del mundo. En efecto, ambos denuncian el círculo al que está sometida la fenomenología en cuanto a que supone la existencia del mundo mientras

1. El presente artículo es el resultado de una investigación desarrollada a lo largo de dos etapas. La primera tiene lugar en la Universidade Estadual de Campinas, Brasil, durante 2008 en el marco de una Pasantía de Investigación abocada a la indagación de la relación de Gilles Deleuze y Edmund Husserl, financiada por CONICYT (Comisión Nacional de Investigación Científica y Tecnológica), Chile. Parte de sus resultados han sido presentados en la ponencia titulada Deleuze e Merleau-Ponty en el "Congresso Centenário Merleau-Ponty", Universidade Federal do Paraná, Septiembre de 2008. La segunda se desarrolla durante 2009 en la École Normale Supérieure de Lyon, Francia, en el marco de la Investigación Doctoral abocada a dilucidar la relación de Deleuze con Leibniz, financiada por el Proyecto MECESUP-UCH 0602 (Programa para el Mejoramiento de la Calidad de la Educación Superior - Universidad de Chile). 2. Doctorando en Filosofía por la Universidad de Chile. Profesor en la Escuela de Psicología de la Universidad Miguel de Cervantes, Chile. 
intenta dar cuenta de su constitución. Tal es la crítica que dirige Merleau-Ponty a Husserl cuando analiza la suerte de transposición que efectúa desde el sujeto encarnado al trascendental, como la que se produce entre el mundo real y el noema mundo (MerleauPonty, 1964). Por su parte, Deleuze insiste en que la definición trascendental del objeto en la conciencia supone un mundo capaz de dar sustento al objeto cualquiera (objeto $=X$ ) al que ella se encuentra dirigida o intencionada (Deleuze, 1969). En cualquier caso, la relación con el mundo requiere una reformulación para ambos autores a la hora de desarrollar su propio planteamiento.

En Merleau-Ponty dicha reformulación tendrá que ver con pensar la apertura al mundo que hace posible el sentir (o la percepción). Esta apertura lo conducirá a plantear un entrelazamiento profundo entre el sujeto encarnado en el mundo y el mundo: el quiasmo. Es precisamente con motivo de la percepción que Merleau-Ponty descubre que para que ésta sea posible se debe estar con-fundido con el mundo; para percibir es necesario ser perceptible. Todo ello conduce a un pensamiento del horizonte que desdobla al mundo y lo transforma en un ser preñado de pliegues que no cesan de multiplicarlo. Para Deleuze, repensar la relación con el mundo implica destruirla y convertirla en un encuentro intensivo capaz de introducir una bifurcación capaz de hacer del mundo varios mundos de ahí la importancia de su lectura del cuento de Borges, El jardín de los senderos que se bifurcan. De hecho, es en la intensidad y el acontecimiento que involucran el encuentro del sentir que se definen las condiciones para una génesis del mundo (Deleuze, 1969, serie $\mathrm{XVI}$ ). Es decir, el mundo sólo se constituye con motivo del encuentro entre series intensivas heterogéneas - intensidades, ellas mismas insensibles, pero capaces de producir la sensación - capaces de hacer emerger una nueva sucesión organizada. De ahí que, para Deleuze, la constitución del mundo se dé en relación estrecha con una bifurcación (o encuentro) que al mismo tiempo le deshace y destituye. Por esta razón es que analizar su pensamiento acerca de lo que es el mundo requiere recurrir a Leibniz, una forma muy diferente a lo que desarrolla Husserl.

A la hora de establecer relaciones entre Deleuze y Merleau-Ponty, las estrategias pueden ser variadas. Entre ellas reconocemos al menos tres ya desarrolladas de manera incipiente por algunos investigadores.

En primer lugar, se puede recurrir a las declaraciones explícitas que realiza Deleuze en contra de la fenomenología y, particularmente, a las críticas que dedica a Merleau-Ponty. A partir de ello, Alain Beaulieu, ha desarrollado en su libro Deleuze et la phénoménologie un análisis detallado del posicionamiento de la filosofía deleuziana respecto de la fenomenología. Beaulieu repara en que Deleuze rechaza ejes centrales de dicha corriente precisamente cuando se encuentra planteando sus intuiciones centrales. El mérito de la obra de Beaulieu radica precisamente en que desentraña la necesidad que enfrenta Deleuze de hacer explícitas sus diferencias respecto de la fenomenología en términos del modo como se piensa lo trascendental, el mundo y la corporeidad (Beaulieu, 2004, p., 9-16). En efecto, sin el establecimiento de las respectivas distancias sobre tales puntos, la filosofía deleuziana arriesga ser confundida con una suerte de fenomenología desarrollada en el límite en el que la fenomenología se reformula a sí misma ${ }^{3}$. Ahora bien, las virtudes y límites de la obra de Beaulieu coinciden en que proyectan la investigación respecto de dicha relación sobre el campo problemático más inmediatamente cercano a las soluciones o respuestas. Efectivamente, si hay un ámbito donde inclusive las relaciones mismas parecen insostenibles entre Deleuze y la fenomenología, es al nivel de los argumentos que desarrollan respecto del lugar del sujeto, el estatuto del cuerpo y la valoración del origen, entre otros.

Luego, es posible reconocer una estrategia que se propone evidenciar analogías entre el pensamiento de Merleau-Ponty y el de Deleuze. La tarea puede resultar, en efecto, muy fructífera y llegar a reconocer ciertas concordancias temáticas relativas, por ejemplo, respecto al modo de definir

3. Cfr. comentarios de Beaulieu acerca de la fórmula propuesta por Jean Luc Marion en este sentido. La fenomenología coincidiría con el propósito de la filosofía contemporánea en la medida que ella entiende el desarrollo del filosofar como tarea infinita, en cuya teleología se definirían las problemáticas y tendencias más diversas, lo que permitiría, a su vez, pensar en que el propósito del filosofar encuentre todo su sentido en el derrotero de la fenomenología (Beaulieu, 2004, p. 12). 
la filosofía, ${ }^{4}$ la importancia con que concentran esfuerzos en una ontología de lo sensible, ${ }^{5}$ o bien, el modo como realizan sus respectivos acercamientos a ciertos pensadores (destacan entre ellos Leibniz y Heidegger). ${ }^{6}$ Sin embargo, las concordancias o parentescos no garantizan la posibilidad de reconocer vínculos más profundos, de modo que esta tarea podría restringirse nada más que al reconocimiento de semejanzas circunstanciales, ellas mismas ajenas al modo de concebir y ejercitar la filosofía que sustentaba cada autor.

Una tercera posibilidad se vislumbra a partir del modo como entiende cada uno el ejercicio del pensar, en la medida que ambos pensadores demostraron una insistente preocupación por definir el estatuto de la interrogación y el problema filosófico. En el nivel de las soluciones propuestas, una dialéctica vulgar impone la necesidad de recorrer los dos caminos simultánea o sucesivamente, procurando establecer contrastes que en algún momento puedan evidenciar vinculaciones profundas. De hecho, un camino se resuelve como inverso del otro precisamente en la medida que tras cada estrategia es siempre posible situar una disparidad, o bien, una vinculación más profunda. Sin embargo, en el nivel de los problemas, el pensar descubre siempre un trance genético que, previo al establecimiento de las relaciones, hace vibrar las interrogantes de unos en otros. Dicha vibración requiere, de todos modos, ser precisada para diferenciarla del reconocimiento de analogías que es posible establecer entre ambos. De esta manera podemos notar que, mientras la analogía se presenta como el camino abierto por la tentativa de generalización reflexiva que descubre la semejanza interna de las categorías, la resonancia mienta un contacto de expresión mutua en cuyos movimientos es posible reconocer no sólo la materialidad del encuentro, sino también las determinaciones recíprocas que en el plano ideal definen la insistencia de instancias de diferenciación que rigen el desenvolvimiento de las problemáticas centrales para cada autor.

\section{2}

Así pues, el modo como cada uno entiende la filosofía será determinante para ello. Deleuze insiste desde sus primeras obras (Empirisme et subjectivité, 1953; Le Bergsonisme, 1966) en que la filosofía consta de problemas que proporcionan una distribución de las interrogantes y de los conceptos en cuya articulación se condensan las intensidades que recorren al pensar y que vibran en él. Este mismo gesto se repite a lo largo de toda su obra, hasta que encuentra en Qu'est-ce que la philosophie? (1991) la oportunidad de un tratamiento más detallado y sistemático. MerleauPonty, por su parte, destaca en su Phénoménologie de la perception que la filosofía debe ser capaz de elevarse al nivel de la autorreflexión donde pueda dar cuenta de su propia génesis y condiciones que lo vinculan al mundo tematizado. En efecto, es entonces cuando se torna primordial su preocupación por el cuerpo (Merleau-Ponty, 1945, Introduction - IV; Barbaras, 1947, Text. commentés I). Más tarde, en Le visible et l'invisible, la filosofía es definida a partir de un interrogar propio del asombro (entonnement) ante la percepción de la génesis del mundo: ahí donde precisamente las cosas se hacen y deshacen, ahí donde se puede tener experiencia de lo que él denomina ser poroso, carácter esencial del mundo entendido como horizonte (Merleau-Ponty, 1964, p. 138 y ss.). Las intensidades que recorren al concepto, así como el asombro característico de la percepción interrogante, acontecen ambas en una suerte de deslizamiento que evidencia un retardo originario ${ }^{7}$ sea el de la asimetría insoslayable en que se encuentran las entidades larvarias, como sucede en el caso de Deleuze, o sea en el hacerse y deshacerse de las cosas, capaz de constatar la vibración de un pensar que deja ser al mundo y que, como tal, lo descubre en su porosidad, característica de la que nos habla Merleau-Ponty.

Lêtre effectif, présent, ultime et premier, la chose même, sont par principe saisis par transparence à travers leurs perspectives, ne s'offrent donc quà quelqu'un qui voit,

4. Entendida fundamentalmente a partir de la posición de problemas. (Deleuze y Guattari, 1991 , l; Merleau-Ponty, 1964)

5. En el caso de Deleuze resultan determinantes en este sentido obras como Différence et répétition y Logique de la sensation. Mientras en Merleau-Ponty lo son Phénoménologie de la perception y, especialmente L'invisible et l'invisible.

6. Cfr. Deleuze, 1988, p. 37; donde a propósito de la inclusión de la mónada en el mundo el autor se refiere a las relaciones entre Heidegger y Merleau-Ponty. En el caso de éste último las relaciones con Heidegger son particularmente evidentes en sus Notes de cours, 'La philosophie aujourd'hui' (cours de 1958-59).

7. Barbaras, 1997, p. 9. Retardo originario es el término con el cual Barbaras se refiere al modo como la reflexión se encuentra a sí misma siempre fuera de lugar (après-coup) en la vida prerreflexiva. Deleuze piensa el retardo originario a propósito de la repetición característica del fantasma (Deleuze, 1968, pp. 162 y ss). Es importante destacar, de todos modos, que Deleuze recusa la posibilidad de aludir a la originariedad del retardo en tanto éste designa el medio intersticial de la forma pura del tiempo entendida como coexistencia virtual de todos los tiempos. La coexistencia virtual determina las condiciones de la génesis ontológica del mundo, ahora bien, en tanto se trata de un campo de coexistencia intersticial no puede ser pensado como originario, pues nada en él puede atribuirse primordialidad ni real ni trascendental. 
non les avoir, mais les voir, non les tenir comme entre des pinces, ou les immobiliser comme sous l'objectif d'un microscope, mais les laisser être et assister à leur être continué, qui donc se borne à leur rendre le creux, l'espace libre qu'ils redemandent, la résonance qu'ils exigent, qui suit leur propre mouvement, qui donc est, non pas un néant que l'être plein viendrait obturer, mais question accordé a l'être poreux qu'elle questionne et de qui elle n'obtient pas réponse, mais confirmation de son étonnement. II faut comprendre la perception comme cette pensé interrogative qui laisse être le monde perçu plutôt qu'elle ne le pose, devant qui les choses se font et se défont dans une sorte de glissement, en deçà du oui et du non" L'invisible et l'invisible, p. $138{ }^{8}$

Para Merleau-Ponty, la reflexión filosófica tiene por necesidad esencial el retrotraerse al ámbito prerreflexivo en que tiene lugar la pura percepción del mundo y donde corresponde pensar dicha percepción como una interrogante, en cuyo caso las preguntas y el cuestionamiento representan verdaderas resonancias de las instancias genéticas del mundo. Como sabemos, el mundo en Le visible et l'invisible es definido por su porosidad, esto es, por la capacidad que ostenta para presentarse como mundo en cada cosa o, dicho de otro modo, por la capacidad que ostenta cada cosa para abrir nuevos horizontes para el mundo. En Le visible et l'invisible, MerleauPonty llama carne a dicha capacidad o potencia de percepción y presentación del mundo. En efecto, es en tanto que el percibir se encuentra engagé, comprometido, en la carne del mundo que ambos ostentan la potencia de la percepción que representa siempre un acontecimiento enigmático, en la medida que se enfrenta al surgir y desvanecerse mismo de las cosas, a la génesis de mundo. Dicha génesis corresponde precisamente a la instancia en que la percepción se produce en una suerte de deslizamiento por el cual la cosa (en tanto instancia porosa) abre nuevos horizontes de mundo o, si se quiere, expresa el mundo. En la percepción resuena así la potencia de presentación misma del mundo que es la carne. Para ser rigurosos también es necesario hacer una precisión. La presentación debe ser entendida como presencia o haber del mundo (Il y a) de acuerdo con la encarnación misma de la percepción. No corresponde pensar presentación y percepción como separadas pues, en rigor, la percepción es ella una sola $y$ es en tanto que percepción encarnada o percepción de sí, que se da para ser percibida, que se presenta. En este sentido, la potencia de percepción que es la carne no es sino una capacidad para percibir-se conforme a la cual una cosa resuena en las interrogantes que la descubren como confirmación del asombro $y$, como destaca Merleau-Ponty, nunca como respuesta.

\section{3}

En virtud de que desde Merleau-Ponty es posible pensar la percepción encarnada como un percibir-se, corresponde reconocer en ella ciertas resonancias leibnizianas con las que Merleau-Ponty se hace eco de la interpretación heideggeriana de la idea de mónada. De hecho, Heidegger recurre a la idea de mónada para mostrar con mayor claridad la relación de inserción del Dasein en el mundo. Con esta interpretación, se da un nuevo giro a la explicación original conforme la mónada no tendría ventanas, presente en Monadología (Leibniz, 1978, §7). La descripción de Leibniz, de acuerdo con la cual la mónada no sostendría ningún tipo de comercio con las demás ya que contiene en sí misma el principio de desenvolvimiento de lo que le sucede, representa la expresión máxima de la unidad del sistema de armonía preestablecida, de acuerdo con el cual Dios crea cada una de las mónadas en perfecto concierto y de tal modo que éstas no requieran contacto real entre ellas. La alusión a la inexistencia de ventanas viene a coronar así una de las primeras formulaciones del sistema de Leibniz, presente en Discours de Métaphysique, acerca de la idea de substancia individual donde al ostentar ésta el principio interno de lo que le ocurre no requiere más que del contacto con Dios, contacto que representa la capacidad de la mónada para expresar su perfecta armonía con las demás, lo que para Leibniz equivale a que pueda expresar el mundo (Leibniz, 1988, § 13-15).

8. “El ser efectivo, presente, último y primero, la cosa misma, son en principio cogidos en su transparencia, a través de sus perspectivas. No se ofrecen, por tanto, más que a alguien que les ve, no a alguien que les posee, no a alguien que les sostiene como entre las pinzas o que les inmoviliza como si estuvieran bajo el objetivo de un microscopio, sino alguien que les deja ser y asistir a su ser continuo, alguien pues que se limita a darle un hueco, el espacio libre que ellos demandan, la resonancia que ellos exigen y que sigue a su propio movimiento. Espacio que es, por tanto, no una nada que el ser pleno vendría a obturar, sino pregunta acorde con el ser poroso que ella cuestiona y del que ella no obtiene respuesta, sino confirmación en su asombro. Es necesario comprender la percepción como este pensamiento interrogativo que deja ser al mundo percibido en vez de efectuar su posición, antes de que les cosas se hagan y deshagan en una suerte de deslizamiento, más acá del sí y del no." 
Para Heidegger, la ausencia de ventanas expresa la implicación del Dasein en el mundo. Esta vez se trata no de evidenciar la armonía de la creación divina, sino de mostrar cómo la inserción (engagement) de la mónada en el mundo, se produce a partir de una imbricación tal que hace superflua la tesis de exterioridad para mostrar la relación de expresión subsistente entre la mónada y el mundo (Heidegger, 2000, §20e; Merleau-Ponty, 1964,pp. 264, 276 y ss.; Deleuze, 1988, pp. 36-7).

Volviendo al problema de la percepción notamos que, en la mónada, ella no depende de una relación de exterioridad, sino más bien del modo como cada una está inserta en el mundo, es decir, con la perspectiva que tiene de él y con el modo como despliega los acontecimientos compatibles dentro de él (Leibniz, 1988, §13). Así, la distancia, intimidad o exterioridad del mundo dependen de la potencialidad de presentación propia de las cosas, esto es, de su encarnación en el mundo. De ahí que no respondan a las distinciones empíricas de interioridad o exterioridad. Una mónada es interior al mundo en la medida que lo expresa y lo contiene, de ahí que no requiera de ventanas pues ella sale de sí en su propio percibir-se. En este sentido, para Leibniz toda percepción es en el fondo autopercepción en la medida que en cada mónada cabe el mundo (Deleuze, 1988, VII). Así pues desde Merleau-Ponty sería posible pensar en un haber del mundo (II y a) tal que con cada percepción se abran nuevos horizontes, pues, si en cada percepción lo que tiene lugar es al mismo tiempo el percibirse, entonces cada percepción constituye el modo como una cosa (mónada) da cuenta del mundo que lleva consigo, de su carne.

En el caso de Deleuze, la instancia genética que retratamos remite igualmente a una suerte de deslizamiento, pero en este caso el que se produce más bien entre series en cuya disparidad sería posible evidenciar un cierto retardo, si nos valemos de la expresión ya aludida de Barbaras (1997, p. 9). Ahora bien: aquí Deleuze no llama retardo a una vuelta del pensar sobre sus condiciones originarias, sino más bien a lo que por definición no admite originariedad, esto es, a la forma pura del tiempo en que coexisten pasado y futuro, antes y después. Retardo es, pues, la coexistencia virtual de todos los tiempos en el tiempo. Forma pura que Deleuze denomina Aion. ${ }^{9}$ Ciertamente, no es posible aducir que la originariedad en Merleau-Ponty tenga relación con algún tipo de antelación que suponga una sucesión serial en el tiempo. Dicha originariedad precisa todavía mayor análisis. Sin embargo, lo que de momento es claro es que para Deleuze resulta necesario rechazar la adscripción del retardo a la originariedad, en la medida que con él lo que se intenta pensar es la génesis a partir de la incomposibilidad de series entre las cuales resulta imposible aducir una relación de anterioridad o sucesión cualquiera.

La génesis para Deleuze se produce a partir de infinitas series incomposibles que conspiran para articular el mundo (1969, XVI). De esta manera, la génesis misma prescinde de la organización serial al postular una forma pura del tiempo indiferente a las sucesiones. Empero, la necesidad de que en el deslizamiento entre seres dispares se produzca la composición de mundos internamente consistentes, y la disyunción respecto de los que no lo son, genera la emergencia de la serialidad. En efecto, sólo se puede hablar de divergencia en condiciones de regularidad para cada una de las series respecto de las cuales se testimonia cierta divergencia. ${ }^{10}$ Merleau-Ponty lee ya el gesto deleuziano en Bergson. En este caso, se trata de la prescindencia de relaciones espaciales para privilegiar la sucesión de estados compenetrados unos con otros, aquello que Bergson llamará duración (Merleau-Ponty, 1996, pp. 198-209; Bergson, 1970). En efecto, las dificultades que experimenta Bergson para explicar la duración en su Essai sur les donnés immédiates de la conscience tienen que ver precisamente con el abandono del lenguaje relativo a las descripciones espaciales y la necesidad de dar cuenta del transcurso del tiempo en términos que no impliquen suponer las divisiones propias de la extensión.

Deleuze asume un desafío similar cuando procura pensar en la ebullición de mundos mutuamente incompatibles que se produce con motivo del deslizamiento entre instancias dispares que se encuentran. La sucesión se suspende y emerge una instancia de indiscernibilidad que borra las fronteras, pero no porque vuelva todo indiferente o indistinguible, sino porque hace coexistir todos los mundos de una vez bajo la forma pura del tiempo, aquella que define también a partir de la insistencia de pasado y futuro a la 
vez (Aion). Dicha coexistencia representa para Deleuze la instancia genética por excelencia, en la medida que en ella habitan cualidades flotantes en estado puro, esto es, intensidades puras inatribuibles cuya vaguedad les permite cabalgar sobre infinidad de mundos que todavía no son y que probablemente jamás sean, pero que insisten y amenazan el derrotero de las series regulares con cada encuentro (1969, XVII). Toda suerte de sujetos larvarios, de cualidades en estado de emergencia, de sensaciones se concentran en puntos singulares que vibran conforme son atravesados por infinitas intensidades. En la medida que Deleuze piensa la instancia genética del mundo, en los encuentros no requiere aducir originariedad alguna. En este sentido, los encuentros garantizan que toda génesis tenga que ver con un estar entre, lo cual no coincide ni con el dominio de la anterioridad ni con el de la finalidad. Así, cabría decir que las bifurcaciones se dicen de los encuentros. Inexpresables en sí mismos - en tanto abren una indiscernibilidad que hace coexistir en un tiempo, o sea en el del encuentro, todos los tiempos -, los encuentros sólo pueden ofrecerse en el testimonio de una bifurcación que les refrenda a posteriori.

Para Leibniz, la bifurcación representa el ámbito de la voluntad conforme al cual Dios decide crear uno de entre la infinidad de mundos que su entendimiento ha podido concebir. Ahora bien, en tanto los concibe en la eternidad, podría decirse que coexisten en su entendimiento todos los tiempos. Como insinúa JeanClet Martin en su lectura de la relación entre Deleuze y Leibniz, ${ }^{11}$ la postura deleuziana requiere reproducir la infinitud leibniziana al modo de un inconciente poblado de intensidades capaces de producir la incomposibilidad de los mundos. ${ }^{12}$ La infinitud, que para Leibniz se halla radicada en el entendimiento divino, para Deleuze insisteen la miríada de tendencias que se insinúan tras cada percepción de la mónada. Deleuze denomina a tales tendencias intensidades. Se trata de un burbujear infinito, un pulular de instancias larvarias (Borges, 1994) que recorre los cuerpos y que puede resolverse en percepción toda vez que la mónada es capaz de integrar en un punto, es decir, en una perspectiva (en una mirada), las infinitas intensidades que bullen desde sus profundidades. Ahora bien, se trata de infinitud en el caso de las intensidades porque para definirla epistemológica y ontológicamente se precisa de diferenciales que remiten a otros diferenciales sin que la sucesión del discernimiento, ni la de la realidad tenga un punto en el que detenerse. De ahí que la zona de claridad por la cual la mónada define su individualidad flote sobre un fondo obscuro de intensidades indiscernibles que pululan por su cuerpo.

\section{4}

La originariedad del tiempo para MerleauPonty dice relación con una génesis en la que coexisten también tiempos incomposibles y en la que, a su vez, se manifiesta cierta incompatibilidad de los espacios. ${ }^{13}$ Lo denomina en sus notas de trabajo del curso dictado durante 1960 y 1961 como tiempo magma (1996, pp. 206 y ss). Ahora bien, no hay que olvidar que para él dicha incomposibilidad está en relación con las sensaciones y las equivalencias que ellas trazan en la génesis de lo mundano. ${ }^{14}$ Podría pensarse que se trata del tiempo dando cuenta de la carne del mundo, esto es, de su potencialidad para que infinidad de poros - o sea, encarnaciones en las que el mundo se percibe - abran en el mundo nuevos horizontes. Pensar en todos los horizontes a la vez es, ciertamente, hacer concurrir tiempos y espacios incomposibles, pero que sin embargo manifiestan entre sí equivalencias que dotan de consistencia al mundo. Así como para Deleuze el sustrato de la sensibilidad, la intensidad, es siempre la instancia de una concurrencia azarosa (un encuentro en que pulula lo larvario) siempre desarticulante (en tanto difiere ella misma respecto de sí), para Merleau-Ponty la sensación representa la instancia en que las cosas manifiestan su pertenencia al mundo en tanto cada sensación es la equivalencia de

11. Cfr. Martin, Jean-Clet (1993, Chap. Le Jardin aux sentiers qui bifurquent). Martin hace referencia al pasaje de Théodicée (Leibniz, 1900 , § 225) en el cual se indica que todos los mundos imaginados por Dios cohabitan en una misma temporalidad. La variedad de referencias con las que Deleuze constituye la idea de coexistencia virtual reconoce entre sus fuentes más importantes la idea de memoria virtual de Bergson (Deleuze, 1966), así como el principio de continuidad del mundo que postula Leibniz (Deleuze, 1967 / 2002).

12. El infinito representaría en Leibniz una infinitud inconciente que insiste a través de tendencias o intensidades en los cuerpos y no la restitución de un entendimiento divino característico de una cosmología racional. Este punto de vista necesario para la interpretación que va a sostener Deleuze de Leibniz se encuentra ya en Belaval (1962, p. 207-212)

13. A diferencia de Bergson, Deleuze manifiesta una valoración positiva del espacio. No obstante, se manifiesta siempre a distancia de una interpretación del mismo en términos de extensión. En este sentido, desarrolla el mismo gesto bergsoniano de rechazo de la espacialidad concebida por Descartes. Prefiere, pues, la interpretación leibniziana que lo define como spatium pre-extensivo; definición que intenta interpretar en términos de la topología contemporánea de Albert Lautman (Deleuze, 1969, XV).

14. Equivalencias que corresponden al logos tácito de lo mundano: "I'apparition sensible est, en sa singularité même, un principe d'équivalence et donc un segment du logos tacite du monde" ["la aparicion sensible es, en su singularidad misma, un principio de equivalencia y, por tanto, un segmento del logos tácito del mundo"] (Barbaras, 1997, p. 33). 
otras sensaciones. Por ello, la incomposibilidad en la que piensa Merleau-Ponty evidencia el torbellino que habita ese magma, la coexistencia cada vez de todos los tiempos, mas no que tales tiempos sean indistinguibles ${ }^{15}$ ni tampoco que remitan a una desarticulación entre mundos incompatibles. El tiempo magma evidencia así una armonía preestablecida entre los tiempos regida por la equivalencia que ostentan entre sí los quale sensibles. El éxtasis del tiempo (el torbellino, el magma) no refleja en MerleauPonty una desarticulación radical, sino un modo de temporalización conforme al cual se definen las distancias para el tiempo y el espacio. Sus referencias a la incomposibilidad, aunque no muy conocidas, resultan claves para su explicación del modo característico de cohesión espacio temporal. Dicha cohesión no se asienta en encadenamiento alguno, sino en una cohesión en cuyo seno estalla una simultaneidad de espacios y tiempos fragmentarios, ahí donde"chaque heure il est toutes les heures, à chaque saison toutes les saisons", ${ }^{16}$ Como señala luego, es la diferenciación de la carne en el Ser la que produce dicho estallido, estallido que es al mismo tiempo cohesión en tanto constituye la génesis a partir de la cual se proyectan espacios y tiempos. Ahora bien, se trata de una cohesión precisamente en la medida en que la concurrencia incomposible no refrenda una imposibilidad de actualización de la multiplicidad de los mundos (como lo sería para un Leibniz) sino, antes bien, refleja la definición de los lados o perspectivas conforme a los que se distribuyen espacio y tiempo sin dejar de estar en la totalidad. Al igual que "comme chaque côte de la chaise est toute la chaise", ${ }^{\prime 7}$ cada tiempo es todos los tiempos y está en todos ellos.

Ciertamente, es el gesto fenomenológico el que lo mantienemásfuertementealejado deDeleuze, puesa pesar de pensar en una concurrencia incomposible de los tiempos su objeto es dar cuenta del transcurso de la descripción fenomenológica sin recurrir a la sucesión husserliana o bergsoniana ni tampoco a la apodicticidad del Cogito. De hecho, Husserl llega a proyectar cierta infinitud constituyente en la descripción fenomenológica en tanto sus objetos a describir estarían radicados en una pasividad (de la cultura o del hábito persistiendo en la conciencia) que haría de toda descripción una tarea infinita. ${ }^{18}$ Merleau-Ponty prescinde del esquema serial y se aboca a pensar la descripción a partir de aquella forma temporal que describe como magma, concurrencia en cada parte de todas las partes.

\section{5}

C'est ainsi que la musique doit rendre sonores des forces insonores, et la peinture, visibles, des forces invisibles ${ }^{19}$

(Deleuze, 1981, p. 39).

A pesar de la distancia constatada en principio entre los quale de sensación en los que piensa Merleau-Ponty la equivalencia del mundo (y en que se insinúa una cierta armonía preestablecida) y las intensidades comprendidas como diferencias internas, insensibles ellas mismas, pero que movilizan el encuentro con las sensaciones, es preciso extenderse más en este punto y constatar ciertas resonancias.

Para Deleuze, la sensibilidad se funda en intensidades cuya constitución paradójica (intensidad es lo que difiere respecto de sí) impide que sean ellas mismas sensibles. La intensidad de la sensación es la sensación en sí misma y, como tal, no es sensible aunque sus efectos puedan serlo. Si se quiere, Deleuze descubre la intensidad como la condición de posibilidad de la sensación y como tal condición no puede ser ella sentida. Por ello la denomina como aistheteonoserdelosensible("être dusensible")yevita retrotraerla al principio aristotélico de la sensación, el aistheton, en tanto éste representa la referencia de la sensación a su principio de actualización que en el

15. Cfr. la insistencia de Merleau-Ponty por separar esta incomposibilidad de lo indistinguible en Notes de Cours, 'L'ontologie cartésienne et l'ontologie d'aujourd hui', p. 198 y ss.

16. ".. a cada hora son todas las horas, a cada estación son todas las estaciones" (Merleau-Ponty, 1996, p. 200)

17. "... como cada lado de la silla es toda la silla". Descripción eminentemente fenomenológica que recuerda las descripciones de Husserl. La unidad de la intencionalidad permite que, en la descripción noemática, los lados del fenómeno estén intencionalmente dirigidos hacia los demás y con ello a la totalidad del fenómeno en cuestión. Sin embargo, cabe destacar en este punto que MerleauPonty desea mantenerse cuidadosamente al margen de la idea de sucesión que entraña este tipo de descripción en Husserl (y de modo similar en Bergson). Su idea de tiempo magma alude precisamente a ello, se trata de una equivalencia de las cualidades que permite medir la distancia en el mundo. La diferencia en el mundo no dependerá así de la sucesión en la descripción, sino de la equivalencia de las cualidades manifiestas por los costados del fenómeno y entreveradas en el magma.

18. La forma temporal de acuerdo con un esquema de descripción, inadecuada pero apodíctica, se encuentra ya desde las Lecciones de fenomenología de la conciencia interna del tiempo (Husserl, 2002: Cap. II). Esta problemática es reiterada en las Meditaciones cartesianas (Husserl, 1986, §9) y sintetizada como la forma del desarrollo histórico en Krisis (Husserl, 1991, \$6, 26, 27).

19. "Es así que la música debe volver sonoras las fuerzas insonoras; y la pintura, visibles las fuerzas invisibles". 
caso de Aristóteles es la cosa sentida. Para Deleuze, el aistheton no puede ser más que el efecto de un principio que rige el devenir actual de la sensación, la intensidad o ser de lo sensible (Deleuze, 1968, V; 1981, VI, VIII). La intensidad mienta una diferencia imperceptible e infinitamente pequeña que recorre a los cuerpos forzándolos a sentir. De ahí que lo sensible corresponda a la integración de infinitas intensidades llevadas al límite.Tal como acontece con la percepción, en Leibniz es la concurrencia de infinitas tendencias e insinuaciones inconcientes la que produce en el límite la percepción. En este caso, el límite designa el umbral que da paso a la conciencia sensible (a la sensibilidad), pero al mismo tiempo indica el umbral que permite el encuentro entre intensidades. En dicho encuentro, las intensidades, que van hasta su propio límite, resuenan unas en otras, haciendo posible el sentir. De modo derivado, el encuentro permite explicar también la relación entre lo sensible y el 'sintiente'. En este caso se trata de figuras o formas forzadas a salir de sí, llevadas al límite, y que en el umbral experimentan la resonancia del encuentro de intensidades: sienten. A pesar del guiño dirigido a la fenomenología con motivo de la descripción de este encuentro (Deleuze, 1981 , p. 27), es preciso seguir con cautela las palabras de Deleuze. Para él, la sensación mienta el resultado de la acción de las fuerzas intensivas llevadas al límite que como tales obligan a la deformación del cuerpo, lo que equivale a decir que obligan a la destrucción del principio de actualización de las sensaciones, para desplegar en el cuerpo una zona dispuesta para encuentros y experiencias inéditos. Esto constituye precisamente una virtualización del cuerpo, que implica la pérdida de su forma y organización: un cuerpo-sin-órganos. Deleuze tematiza todo aquello en Francis Bacon. Logique de la sensation, a partir de fórmulas nunca antes utilizadas en su obra. Llama la atención, por ejemplo, que se refiera a la deformación del cuerpo como una salida del mismo hacia la carne. El cuerpo-sin-órganos es tematizado, en este sentido, en Logique de la sensation como carne.

La cercanía con Merleau-Ponty es evidente en este nivel, mas no por la simple concurrencia terminológica. Con carne Deleuze designa el campo de fuerzas indomables que despliegan toda suerte de tentativas sobre el cuerpo para obligarlo a salir de sí, a perder su forma, y a experimentar el encuentro característico de la sensación. Lo expresa también diciéndonos que la actividad estética (presente en la música o en la pintura) tiene por objeto hacer sensibles aquellas fuerzas o intensidades insensibles; gracias a ello, el cuerpo que siente se eleva de alguna forma hacia su condición, aquella que, permitiéndole los encuentros sensibles, es ella misma insensible; la carne (1981, VI). Condición que Merleau-Ponty piensa en términos de una potencia del sentir como reverso mismo de la sensibilidad y en cuanto tal resulta ella misma insensible. La carne para él es precisamente la potencia invisible de la visibilidad, esto es, la posibilidad del sentir que como tal hace concurrir al mundo a su mutua presentación. La fuerza en definitiva que rige tras la percepción. Ahora bien, corresponde ser cuidadosos para no producir equivalencias apresuradas entre nuestros pensadores. No hay que olvidar nunca que la potencia de la carne fuerza cierta pregnancia de las cosas en el percipiente, de manera tal que lo que fuerza es la imposición de una forma en él. La carne es así el principio de actualización de las formas que rigen el sentir. Sentir para Merleau-Ponty es la percepción de las formas, sólo que en una modalidad que evidencia la presencia misma del principio de actualización de tales formas. Invisible él mismo, es potencia que guía toda suerte de relaciones en el mundo y que hace del mundo, mundo. Esto es, que refrenda su presencia, el hay (Il y a) conforme las cosas se perciben gracias a la potencia de percibirse, a su estar encarnadas.

A pesar de la distancia entre la ontología de la sensación de Merleau-Ponty, que lleva hasta sus límites la imposición de la forma (en tanto pregnancia del mundo en el percipiente), pensando con ello la potencialidad de la carne ${ }^{20}$ y la que esboza Deleuze, en cuya tentativa de virtualización procura hacer de la carne no el principio de la forma, sino de su disolución, es posible leer ciertas resonancias importantes a propósito de poder pensar las condiciones del sentir.

La importante tarea impuesta a una filosofía de esta índole es pensar la disolución del abismo que la tradición ha colocado entre sensibilidad y entendimiento. ${ }^{21}$ Ello exige pensar una relación entre las potencialidades del sentir y la emergencia de la idea o del concepto. Podría sostenerse que el concepto queda librado nuevamente a su suerte para convertirse en idea en la medida que no tiene que dar cuenta de la suposición de un mundo como sustento de objetos cualesquiera. El entendimiento, en tanto, deja de ser esa facultad 
rectora de la sensibilidad que la supone como referencia de sentido. ${ }^{22}$ Otra suerte de relaciones se producen entre ellas tales que fuerzan a pensar en una percepción interrogante que evidencia las perplejidades que implica la presentación del mundo (Merleau-Ponty), relaciones que llevan también a reconocer un vínculo secreto y directo entre la intensidad y la idea de modo tal que la primera constituye una potencia de virtualización capaz de hacer coexistir consistentemente el sobrevuelo universalizante de lo que puede estar en varios mundos incomposibles, a la vez que la singularidad de lo que difiere respecto de sí (Deleuze).

Una resonancia queevidencia contemporaneidad no de las datas, sino de los problemas. Un problema que persiste en un autor y que hace estallar tentativas de resolución en el otro. Una interrogante que aquí produce la percepción y que allá obliga a pensar a partir de la experiencia extática de lo que no puede ser sentido. La resonancia no mienta un procedimiento de configuración de contrastes o vinculaciones, no dice relación ni con el movimiento que conduce a la contradicción, ni con la generalización que lleva hasta la analogía. Para ser más exactos, se trata del procedimiento de vice-dicción en el que se manifiesta una diferencia más radical que la propia contradicción y una resonancia secreta más íntima que la de la analogía. La vice-dicción mienta, como lo señala Christiane Frémont, ${ }^{23}$ un procedimiento de homogonía distinto de la homología, en tanto constituye la relación que se establece entre una clase y un atributo inesencial característico de una clase alterna. Consiste, como lo señalara Deleuze, en la definición de lo esencial a partir de lo inesencial (Deleuze, 1967; Deleuze, 1968, p. 245), procedimiento siempre necesario para Leibniz, por medio del cual le era posible explicar la inclusión del mundo en la mónada - que por lo mismo incluye infinitos atributos inesenciales en la definición de su esencia. En este sentido constituye la resolución lógica de los problemas relativos a la resonancia entre mónadas que supone la armonía preestablecida. ${ }^{24}$

De esta manera, la exploración de las condiciones genéticas del sentir en cada autor refrenda otro tipo de sensación, esta vez en el nivel donde se define el estatuto de los problemas para cada filósofo. Una sensación aquí es motivo de una idea allá, un concepto allá produce una miríada de experiencias de este lado. No es posible reducir dicho contacto a un mero diálogo intelectual; es realmente una intensidad la que los recorre en su mutua vibración. A su vez, es preciso no confundir el contacto con una suerte de comunidad espiritual. En este caso no cabe la analogía monadológica, pues se trata innegablemente de planteamientos incomposibles. Para decirlo de forma más precisa: es en tanto incomposibles que los planteamientos de Merleau-Ponty y Deleuze no cesan de resonar unos en otros, refrendando la apertura de un campo problemático que los dota en su diferencia de una íntima contemporaneidad, cual si se tratase de la contemporaneidad de los tiempos que uno y otro tratan de dilucidar ante la perplejidad del mundo. En efecto, como seguramente lo pensaron cada uno de ellos, la idealidad del pensar vibra incesantemente en un sentir que, antes de volverse sensible, se resuelve en idea. Sea que provengan de la cercanía y del rigor de una deducción lógica interna, o de parajes lejanos, el universo entero conspira en cada pensador dirigiéndolo en cada una de sus proposiciones. Los pensamientos de Merleau-Ponty y de Deleuze, innegablemente incomposibles, se reconocen, en suma, en un entrecruzamiento tan casual como las sensaciones que aspiran a comprender, pero a su vez tan necesario como los afectos que los hacen vibrar hasta hacer salir de ellos aquella novedad, aquella idea que brilla por sobre el fondo obscuro de sensaciones que no llegan jamás a actualizarse, por sobre la pura potencia que es carne del mundo.

20. "Le sujet est fait de la même texture que le monde et il ne peut l'atteindre que parce qu'il est enveloppé par lui" ["El sujeto está hecho de la misma textura que el mundo, mundo que el sujeto no podría alcanzar en tanto está envuelto en él"] Merleau-Ponty, 1964, p. 277)

21. Por cierto, hacemos alusión a la Crítica de la razón pura de Kant, particularmente a los pasajes referidos al esquematismo de los conceptos puros del entendimiento.

22. Coincidencia importante entre Deleuze y Merleau-Ponty, a propósito de la necesidad de la filosofía reflexiva de hacer referencia a objetos posibles (objeto X) en un mundo como referencia de la intencionalidad y particularmente como sustento de los noemas. Necesidad que ambos critican muy duramente (Merleau-Ponty, 1964, pp. 276 y ss.; Deleuze, 1969:, XIV).

23. Cabe diferenciar la lectura de la declaración leibniziana que realizan Anne-Marie Roviello y Christiane Frémont. Roviello estima que se trata lisa y llanamente de un procedimiento analógico (Roviello, 2006), mientras que Frémont complejiza la cuestión aludiendo al mecanismo que Deleuze denomina de vice-dicción (Deleuze, 1968, pp. 245-247; 1969, XVI; 1988, V; Frémont, 2003, p. 34).

24. Conforme a la tesis central de Frémont en su libro Singularités, individus et relations dans le système de Leibniz, se trata de lo que permite el paso de la mónada a la monadología, esto es, del individuo singular al sistema de armonía preestablecida, garante de la perfecta relación entre los individuos (Frémont, 2003, Préface). 


\section{Fuentes bibliográficas}

Barbaras, Renaud (1997). Merleau-Ponty. Paris: Ellipses.

Beaulieu, Alain (2004). Gilles Deleuze et la phénoménologie. Mons (Belgique): Sils Maria éditions.

Belaval, Yvon (1962). Leibniz. Initiation a sa philosophie, Paris: Vrin.

Bergson, Henri (1970). Essai sur les donnés immédiates de la conscience. Paris: PUF.

Borges, Jorge Luis (1994). "El jardín de los senderos que se bifurcan"' En: Fictions (éd. bilingue préparée par Roger Caillois). Paris: Gallimard.

Deleuze, Gilles (1966). Le Bergsonisme. Paris: PUF.

Deleuze, Gilles (1967) "La méthode de dramatisation". En: (2002) Lîle déserte et autres textes. Minuit, 2002, p. 127-153.

Deleuze, Gilles (1968). Différence et répétition. Paris: PUF.

Deleuze, Gilles (1969). Logique du sens. Paris: Minuit.

Deleuze, Gilles (1981). Francis Bacon. Logique de la sensation. Paris: Éditions de la différence.

Deleuze, Gilles (1986). Foucault. Minuit, 1986.

Deleuze, Gilles (1988). Le Pli. Leibniz et le baroque. Paris: Minuit. Logique

Deleuze, Gilles y Félix Guattari (1991). Qu'est-ce que c'est la philosophie? Paris: Minuit.

Frémont, Christiane (2003). Singularités, individus et relations dans le système de Leibniz, Paris: Vrin.

Heidegger, Martin (2000). Los problemas fundamentales de la fenomenología. Madrid: Trotta.
Husserl, Edmund (1986). Meditaciones Cartesianas. Madrid: Tecnos.

Husserl, Edmund (1991). La Crisis de las ciencias europeas y la fenomenología trascendental. Barcelona: Editorial Crítica.

Husserl, Edmund (2002) Lecciones de fenomenología de la conciencia interna del tiempo. Madrid:Trotta.

Leibniz, G.W. (1900). "Théodicée". En: Fuvres philosophiques de Leibniz (éd. Par Paul Janet), Paris: Librairie Félix Alcan, pp. 1-369.

Leibniz, G.W. (1978). Monadologie (éd. Emile Boutroux). Paris: Librairie Delagrave.

Leibniz, G.W. (1988). Discours de métaphysique (éd. Georges Le Roy). Paris: Vrin.

Martin, Jean-Clet (1993). Variations. La philosophie de Gilles Deleuze. Paris: Biblioteque Scientifique, Payot.

Merleau-Ponty, Maurice (1945). Phénoménologie de la perception. Paris: Gallimard.

Merleau-Ponty, Maurice (1964). L'invisible et l'invisible. Paris: Gallimard.

Merleau-Ponty, Maurice (1996). Notes de cours 1959-1961. Paris: Gallimard.

Roviello, Anne-Marie (2006) "La communauté des singuliers, entre harmonie préétablie et libre institution". En: Timmermans, Benoît. Perspective Leibniz, Whitehead, Deleuze. Paris: Vrin, pp. 103124 